\title{
Potencial da desmetilação da lignina na produção de adesivo para colagem de lâminas de madeira
}

\author{
Fabiana Paiva de Freitas ${ }^{1 \star}$, Márcia Silva de Jesus ${ }^{1}$, Benedito Rocha Vital ${ }^{1}$, Tais Regina Lima Abreu Veiga ${ }^{2}$, \\ Mariana Fonseca Xisto ${ }^{1}$
}

${ }^{1}$ Universidade Federal de Viçosa (UFV), Viçosa, Minas Gerais, Brasil.

${ }^{2}$ Universidade Federal de Lavras (UFLA), Lavras, Minas Geras, Brasil.

\begin{abstract}
RESUMO A lignina, maior fonte natural de polímero aromático fenólico, apresenta grande potencial para ser utilizado na síntese de novos adesivos, desde que sua reatividade seja alterada com a modificação de sua estrutura química. Diante disso, o objetivo do trabalho foi analisar a resistência ao cisalhamento na linha de cola da madeira de Eucalyptus sp., a partir da utilização de adesivos produzidos com lignina Kraft desmetilada com ácido clorídrico e fenolada. Foram utilizados seis tipos de adesivos, o comercial fenol-formaldeído, o sintetizado no Laboratório de Painéis e Energia da Madeira, o adesivo de lignina fenolada e três adesivos preparados com lignina desmetilada com ácido clorídrico no volume de $40 \mathrm{ml}, 60 \mathrm{ml}$ e $80 \mathrm{ml}$. Os adesivos foram caracterizados e utilizados para a colagem de lâminas, que foram submetidas ao teste de resistência ao cisalhamento por tração. Foi observado que os corpos-de-prova colados com o adesivo de lignina desmetilada (HCl-60ml) atingiram valores de resistência $(5,18 \mathrm{MPa})$ superiores aos demais tratamentos. Dessa forma, os adesivos compostos com lignina desmetilada com ácido clorídrico e fenolada apresentam potencial de uso na fabricação de adesivos para colagem de lâminas de madeira.
\end{abstract}

Palavras-chave: reatividade; biopolímero; estrutura química.

\section{Potential of lignin demethylation in the production of adhesive for bonding of wood veneers}

\begin{abstract}
Lignin, most natural source of phenolic aromatic polymer, has a great potential to be used in the synthesis of new adhesives, since their reactivity is changed with the modification of its chemical structure. Thus, the objective of this study was to analyze the shear strength in the glue line of Eucalyptus sp., from the use of adhesives produced with lignin Kraft demethylated with hydrochloric acid and phenolated. Were used six types of adhesives, phenol-formaldehyde commercial, synthesized in panels Laboratory of Wood Energy, phenolated lignin adhesive and three prepared adhesives with demethylated lignin with hydrochloric acid in volume of $40 \mathrm{ml}, 60 \mathrm{ml}$ and $80 \mathrm{ml}$. The adhesives were characterized and used for bonding blades, which were subjected to tensile shear resistance test. It was observed that the specimens bonded with the demethylated lignin adhesive $(\mathrm{HCl} 60 \mathrm{ml})$ reached resistance values $(5,18 \mathrm{MPa})$ higher than the other treatments. Thus, the adhesive compounds with lignin demethylated with hydrochloric acid and phenolated have a potential use in the manufacture of adhesives for bonding wood veneers.
\end{abstract}

Keywords: reactivity; biopolymer; chemical structure.

\section{Introdução}

Dentre os principais componentes da madeira, a lignina é o segundo composto orgânico mais abundante, dispondo de uma estrutura amorfa e por ser de origem renovável, é considerada a maior fonte natural de polímero aromático fenólico (CARROTT et al., 2007), com grande potencial para ser utilizada na síntese de novos materiais poliméricos (TEJADO et al., 2007; CAVDAR et al.,2008).

Há uma grande quantidade de lignina gerada pelas indústrias de papel e celulose durante todo ano, principalmente a Kraft, entretanto, devido ao seu baixo valor agregado, quase toda a lignina gerada é queimada para geração de energia e recuperação de produtos químicos e 
menos de 5\% da gerada pelo mundo estão sendo usada para outra finalidade, tais como materiais biodispersantes, surfactantes e adesivos para madeira (GOSSELINK et al., 2004; LORA et al., 2002; LEE et al., 2002).

O interesse pela lignina, como uma alternativa para substituir o fenol nos adesivos comerciais de fenolformaldeído, tem aumentado ao longo dos últimos anos. Vários estudos buscam diferentes métodos para reagir à lignina com o fenol e com o formaldeído para formar polímeros. Em tal aplicação a lignina serve tanto como um agente complementar quanto um substituto do fenol. $\mathrm{O}$ uso direto da lignina em adesivos comerciais de fenolformaldeído requer alto tempo e temperatura de prensagem devido a sua baixa reatividade. Portanto, o uso da lignina não modificada não é comercialmente interessante para esta aplicação (HU et al., 2011).

Existem diferentes categorias para melhorar a reatividade da lignina e uma delas é a modificação de sua estrutura química para aumentar o potencial de reatividade dos seus sítios de ligações. Os métodos mais estudados dentro desta categoria são a desmetilação e fenolação da lignina (HU et al., 2011).

Esse biopolímero possui reatividade dos sítios de ligações mais baixo quando comparado ao composto fenol. Sabe-se que o potencial de reatividade dos grupos hidroxilas aromáticas da lignina Kraft são bloqueadas pelos grupos metoxílicos. Se tais grupos são removidos, em seguida, o material resultante teria mais grupos fenólicos livres e, consequentemente, se tornaria mais reativo (THAKUR et al., 2014). Com a desmetilação, os grupos metoxílicos $\left(-\mathrm{OCH}_{3}\right)$ das posições orto e/oupara, da estrutura aromática da lignina são substituídos pelos grupos hidroxílicos $(-\mathrm{OH})$ e sua reatividade aumenta.

A lignina Kraft também pode ser tratada com o fenol na presença de solventes orgânicos, como metanol ou etanol, para a síntese de adesivos para madeira, sendo esse processo chamado de fenolação (EFFENDI et al., 2008). Durante o processo de fenolação, a lignina é termicamente tratada com o fenol e ocorre a sua condensação ao anel aromático e na cadeia lateral. Durante esse processo, ocorre a clivagem das ligações éter, fazendo com que diminua o seu peso molecular. Como resultado da fenolação, obtém-se um material mais reativo, o que melhora seu uso na formulação e síntese de adesivos (GHAFFAR; FAN, 2014; QIAO et al., 2015).

Diante disso, o objetivo do trabalho foi analisar a resistência ao cisalhamento na linha de cola da madeira de Eucalyptus sp., a partir da utilização de adesivos produzidos com lignina Kraft desmetilada com ácido clorídrico e fenolada, para fins estruturais.

\section{Material e Métodos}

\section{Descrição dos adesivos}

Foram utilizados seis tipos de adesivos, o comercial fenolformaldeído, o sintetizado no Laboratório de Painéis e Energia da Madeira, o adesivo de lignina fenolada com 45\% de lignina e $55 \%$ de fenol, e três adesivos preparados com lignina desmetilada com ácido clorídrico no volume de $40 \mathrm{ml}$, $60 \mathrm{ml} \mathrm{e} 80 \mathrm{ml}$ de ácido.

Os adesivos sintetizados no Laboratório de Painéis e Energia da Madeira e o adesivo de lignina fenolada foram preparados de acordo com Khan; Ashraf; Malhotra (2004). A fenolação da lignina foi preparada utilizando $45 \mathrm{~g}$ de lignina e $55 \mathrm{~g}$ de fenol, totalizando uma massa de $100 \mathrm{~g}$ de adesivo.

\section{Desmetilação da lignina Kraft}

A lignina foi fornecida pela empresa Suzano Papel e Celulose, com teor de cinzas e umidade igual a 1,5\% e 5\%, respectivamente, proveniente do processo Kraft da madeira de Eucaliptus sp. A determinação do teor de cinzas foi realizada segundo a norma da NBR $8112($ ABNT, 1986) e para o teor de umidade a norma ABNT NBR 7190 (ABNT, 1997).

Para a reação de desmetilação, as misturas foram preparadas em balões volumétricos de $250 \mathrm{ml}$, utilizando 38,5 $\mathrm{g}$ de lignina Kraft dissolvidas em volumes de 40, 60 e $80 \mathrm{ml}$ de 
ácido clorídrico (38\%). A mistura reacional foi aquecida em chapa $\left(100^{\circ} \mathrm{C}\right)$ e mantida sob agitação magnética em refluxo durante 40 minutos. Após as reações, a camada orgânica foi lavada com água deionizada e seca em estufa $\left(40^{\circ} \mathrm{C}\right)$, (Adaptado de SANTOS et al., 2003). O processo de desmetilação está ilustrado na equação geral, como mostrado na Figura 1.

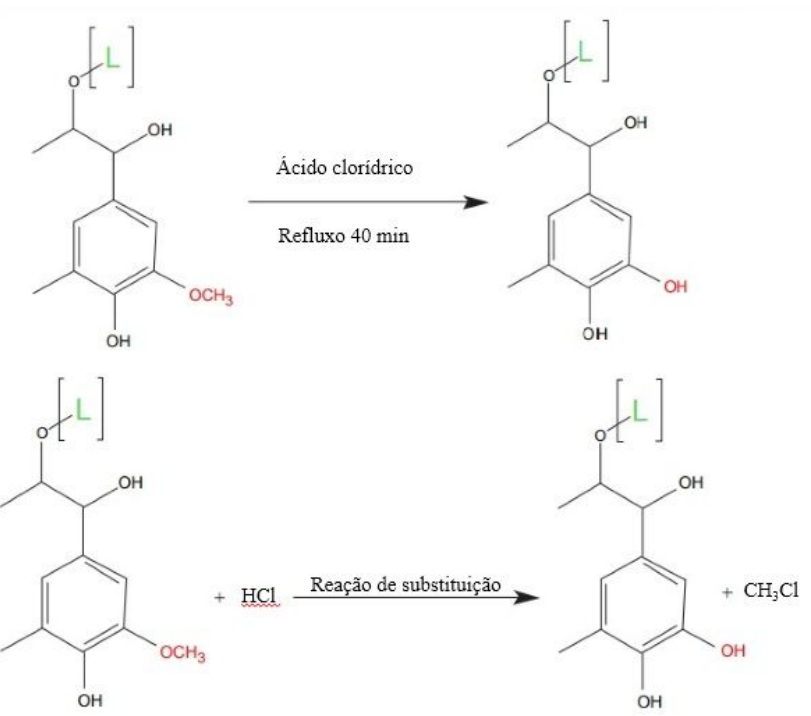

Figura 1. Mecanismo de reação para o processo de desmetilação ilustrando a substituição da metoxila pelo grupo hidroxila em condições de reação otimizadas, em que "L" indica a molécula de lignina. Fonte: Adaptado de Ferhan et al, 2013.

Figure 1. Reaction mechanism for the demethylation process illustrating the methoxyl substitution of hydroxyl group at optimized reaction conditions, where "I" indicates the lignin molecule. Fonte: Adaptado de Ferhan et al, 2013.

\section{Síntese do adesivo de lignina desmetilada}

Após a desmetilação, a lignina $(45 \% \mathrm{~m} / \mathrm{m})$ foi submetida ao processo de fenolação (fenol 55\% m/m), em banho-maria (60 ${ }^{\circ} \mathrm{C}$ ) durante 1 hora. Em seguida o adesivo foi preparado utilizando a lignina desmetilada e fenolada (48,45 g), formaldeído (81,08 g), metanol 50\% (20 g) e hidróxido de sódio $50 \%(4,8 \mathrm{ml} \times 3)$. Os reagentes foram adicionados em balão de fundo chato com 2 entradas e levados para a chapa aquecedora $\left(85^{\circ} \mathrm{C}\right)$ e mantidos sob agitação até o refluxo. Após o refluxo foi contabilizado 17 minutos e, posteriormente, adicionado 4,8 $\mathrm{ml}$ de $\mathrm{NaOH}$ (50\%), controlando a temperatura da reação $\left(85^{\circ} \mathrm{C}\right)$ durante 35 minutos. Depois desse intervalo foi adicionado a última carga de $\mathrm{NaOH}(50 \%)$ e aguardado mais 65 minutos para finalizar a reação (adaptado de FERREIRA, 2017).

\section{Caracterização dos adesivos}

A determinação do teor de sólidos foi adaptada da norma ASTM-D-1518-60 (1994), utilizando 2 g de adesivo. Para cada adesivo, foram feitas três repetições. O teor de sólidos foi calculado por gravimetria (base seca).

A viscosidade do adesivo foi medida a $25{ }^{\circ} \mathrm{C}$, utilizando um viscosímetro Thomas Stormer, com $150 \mathrm{ml}$ de adesivo, em três repetições.

Para a determinação do tempo de gelatinização, aproximadamente $1,0 \mathrm{~g}$ de adesivo foi colocado em um tubo de ensaio $(15,0 \times 2,0 \mathrm{~cm})$ e mergulhados em um banho de glicerina a $120{ }^{\circ} \mathrm{C}$. A agitação dentro do tubo de ensaio foi feita manualmente com um bastão de cobre. Para cada amostra, o tempo foi contabilizado a partir da imersão no banho de glicerina até o endurecimento parcial do adesivo, verificado quando este ofereceu maior resistência ao giro do bastão.

O Potencial Hidrogeniônico $(\mathrm{pH})$ foi determinado com o pHmetro modelo $D$-22. Para cada adesivo foram realizadas três repetições.

\section{Ensaio de cisalhamento na linha de cola}

Foram produzidas lâminas de madeira de Eucalyptus sp., 6 anos de idade, com dimensões de $100 \times 400 \times 6 \mathrm{~mm}$ de largura, comprimento e espessura, respectivamente. Após a amostragem, as lâminas foram secas em estufa, até atingir umidade de $8 \%$, e coladas com os adesivos do tipo comercial fenol-formaldeído, comercial, sintetizado, fenolado, desmetilado com 40, 60 e $80 \mathrm{ml}$ de $\mathrm{HCl}$, utilizando 6 minutos de pré-prensagem, 8 minutos de prensagem, a uma temperatura de $170^{\circ} \mathrm{C}$ e pressão de $12 \mathrm{kgf} / \mathrm{cm}^{2}$. A colagem foi realizada aplicando-se uma gramatura de $200 \mathrm{~g} / \mathrm{m}^{2}$ de adesivo 
nas lâminas e espalhadas com um pincel, até cobrir toda a superfície, para posterior prensagem. Em seguida, foram confeccionados 10 corpos-de-provas por tratamento, cujas dimensões estão apresentadas na Figura 2, e submetidas ao teste de resistência ao cisalhamento por tração de acordo com a ASTM D-2339 2008 (2011).

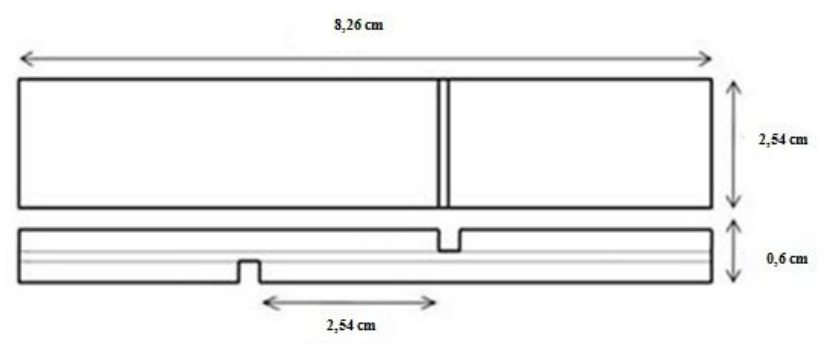

Figura 2. Corpo-de-prova confeccionado para o ensaio de cisalhamento na linha de cola.

Figure 2. Test piece made for the shear test in the glue line.

\section{Delineamento Estatístico}

O experimento foi analisado segundo o delineamento estatístico inteiramente casualizado (DIC). Foram considerados: seis formulações de adesivos ( $\mathrm{HCl}-40 \mathrm{ml}, \mathrm{HCl}$ - 60ml, HCl - 80ml, lignina fenolada, adesivo comercial, adesivo sintetizado em laboratório) e quatro juntas de lâminas coladas por formulação, com total de $n=10$ (dez corpos de prova por junta de lâmina colada), totalizando 240 unidades amostrais. Os dados foram submetidos à análise de variância (ANOVA) pelo teste $\mathrm{F}$, sendo as médias comparadas entre si pelo teste Tukey, adotando-se 5\% de significância. Os dados foram processados utilizando o programa estatístico Sisvar.

\section{Resultados e Discussão}

\section{Caracterização dos adesivos}

Na Tabela 1 estão representados os valores médios do teor de sólidos, viscosidade, $\mathrm{pH}$ e tempo de gelatinização dos diferentes tipos de adesivos. A análise de variância mostrou que houve diferença significativa entre os adesivos para todas as análises realizadas.
Tabela 1. Valores médios do teor de sólido (\%), Viscosidade, pH e Tempo médio de gelatinização dos adesivos.

Table 1. Mean values of solid content, viscosity, $\mathrm{pH}$ and time gelatinization of the adhesives.

\begin{tabular}{ccccc}
\hline Adesivos & $\begin{array}{c}\text { Sólidos } \\
\text { (\%) }\end{array}$ & $\begin{array}{c}\text { Viscosidade } \\
\text { (cP) }\end{array}$ & pH & $\begin{array}{c}\text { Gel time } \\
(\mathrm{s})\end{array}$ \\
\hline $\mathrm{HCl}-40 \mathrm{ml}$ & $46,2 \mathrm{bc}$ & $450,0 \mathrm{~b}$ & $10,7 \mathrm{~b}$ & $101 \mathrm{~d}$ \\
$\mathrm{HCl}-60 \mathrm{ml}$ & $45,9 \mathrm{c}$ & $146,7 \mathrm{e}$ & $10,7 \mathrm{~b}$ & $146 \mathrm{c}$ \\
$\mathrm{HCl}-\mathbf{8 0 m l}$ & $42,9 \mathrm{c}$ & $16,7 \mathrm{f}$ & $10,5 \mathrm{~b}$ & $158 \mathrm{c}$ \\
Lignina & $44,1 \mathrm{c}$ & $206,7 \mathrm{~d}$ & $10,6 \mathrm{~b}$ & $261 \mathrm{~b}$ \\
$\begin{array}{c}\text { Fenolada } \\
\text { Adesivo }\end{array}$ & $51,7 \mathrm{ab}$ & $663,3 \mathrm{a}$ & $11,8 \mathrm{a}$ & $307 \mathrm{a}$ \\
$\begin{array}{c}\text { Comercial } \\
\text { Adesivo }\end{array}$ & $55,8 \mathrm{a}$ & $303,3 \mathrm{c}$ & $11,9 \mathrm{a}$ & $274 \mathrm{~b}$ \\
$\begin{array}{c}\text { Sintetizado } \\
\text { CV (\%) }\end{array}$ & 4,3 & 2,2 & 0,9 & 5,3 \\
\hline
\end{tabular}

CV $(\%)=$ Coeficiente de variação. Médias seguidas pelas mesmas letras não diferem entre si pelo teste Tukey; $\mathrm{p}>0,05$.

Observa-se que o adesivo sintetizado apresentou maior teor de sólidos, diferindo dos adesivos compostos por lignina desmetilada com $\mathrm{HCl}(60$ e $80 \mathrm{ml})$ e do produzido com a lignina

fenolada, que apresentaram os menores teores. Segundo Ferreira (2017), elevados teores de sólidos prejudicam a aplicação do adesivo via aspersão, devido ao aumento da sua viscosidade, assim como também dificultam espalhamento e penetração dos adesivos na madeira.

A menor viscosidade do adesivo desmetilado ( $\mathrm{HCl}-80 \mathrm{ml})$ pode ser explicado devido ao grau de solubilidade da lignina no ácido, visto que, quanto maior o volume de $\mathrm{HCl}$, mais solúvel a lignina tende a ficar e, consequentemente, menos viscosa. Isso pode comprometer a estrutura do biopolímero e, até mesmo, degradá-lo, o que não é desejado no processo de fabricação dos adesivos, por também influenciar na sua resistência.

O adesivo comercial apresentou maior valor médio de viscosidade, enquanto o adesivo desmetilado com $\mathrm{HCl}-80 \mathrm{ml}$ apresentou o menor valor médio. A elevada viscosidade do adesivo comercial, quando comparado aos demais, pode estar relacionado à adição de cargas e extensores, que são aditivos utilizados para a correção da fluidez de adesivos e evitar a super penetração na superfície da madeira, já que isso pode 
gerar uma linha de cola "faminta", que também não é uma característica desejável para um adesivo. $\mathrm{O}$ aumento do peso molecular do adesivo também está diretamente relacionado à sua alta viscosidade, sendo este um processo que ocorre durante a polimerização (PIZZ; MITTAL,1994).

$\mathrm{O}$ pH apresentou pequena variação, de 10,5 a 11,9. Os adesivos preparados com lignina desmetilada e fenolada tiveram pequena redução. A alteração no valor do $\mathrm{pH}$ dos adesivos preparados com lignina desmetilada e fenolada possivelmente, é devido ao processo de produção desses adesivos, em que ácidos são utilizados, ou algum outro componente de característica ácida como, por exemplo, a própria lignina Kraft. Segundo Marra (1992), os valores de $\mathrm{pH}$ para adesivos fenólicos de natureza alcalina variam de 9 a 12, portanto, todas as formulações sintetizadas neste estudo estão dentro da faixa praticada na indústria.

$\mathrm{O}$ adesivo comercial apresentou também maior tempo de gelatinização, enquanto os adesivos a base de lignina desmetilada com $\mathrm{HCl}$ apresentaram menores valores. Notase que a desmetilação da lignina, para a síntese dos adesivos, influencia no tempo entre o início do processo de cura até atingir a fase de máxima elasticidade do adesivo que, possivelmente, foi influenciado pelo aumento da reatividade da lignina. Segundo Pramanik; Karak (2017), o tempo de gelatinização mede a reatividade do adesivo, sendo este um parâmetro que fornece uma noção do tempo necessário para a cura da resina. Essa é uma importante etapa, que afeta diretamente a linha de fabricação de uma empresa de painéis, por exemplo, quanto menor o tempo de prensagem (menor "gel time”), maior a produtividade, além de não aumentar o custo final de produção. Dessa forma, quando comparamos o tempo de gelatinização do adesivo comercial com o tempo médio dos desmetilados $(\mathrm{HCl})$, há uma redução de $56 \%$ do tempo final, que pode ser considerado um valor significativo em termos de eficiência e produção.

\section{Ensaio de cisalhamento na linha de cola}

$\mathrm{Na}$ Tabela 2 encontram-se os valores médios de resistência ao cisalhamento na linha de cola das lâminas coladas, sendo que, estatisticamente, existe, pelo menos, um tratamento que difere dos demais quanto ao valor de resistência.

Pelos resultados apresentados observa-se que os corpos de prova colados com o adesivo produzido com a lignina desmetilada ( $\mathrm{HCl}-60 \mathrm{ml})$ atingiram um valor médio de resistência $(5,18 \mathrm{MPa})$ superior aos demais tratamentos.

A maior resistência das lâminas coladas com adesivos a base de lignina desmetilada (HCL-60ml) pode ser explicado pela alteração na reatividade da lignina, que ocorre a partir da substituição dos grupos metoxílicos $\left(\mathrm{OCH}_{3}\right)$ por grupos hidroxílicos $(\mathrm{OH})$, influenciando o aumento da interação entre os grupos reativos da lignina com o formaldeído, isso resulta em um adesivo mais resistente. Ferhan et al., (2013) confirmam que a conversão do grupo metóxilo por um grupo hidroxilo torna a molécula mais reativa; Os autores comprovaram esse comportamento a partir de análises de cromatografia de exclusão estérica de alta performance (HPSEC) e espectroscopia (FTIR), o que facilita a formação de ligações químicas, a partir do aumento do número de sítios reativos, o que pode levar a produção de adesivos mais resistentes. Outros autores também corroboram com essa idéia (MEISTER, 2000; HU et al., 2011; HU et al., 2014).

Tabela 2. Valores médios da resistência ao cisalhamento das lâminas coladas dos adesivos.

Table 2. Shear strength of the average values of the blades bonded adhesives.

\begin{tabular}{cc}
\hline Tratamentos & $\begin{array}{c}\text { Resistência ao cisalhamento } \\
(\mathrm{MPa})\end{array}$ \\
\hline $\mathrm{HCl}-80 \mathrm{ml}$ & $3,50 \mathrm{c}^{(3,96)}$ \\
Lignina fenolada & $3,64 \mathrm{c}^{(8,63)}$ \\
Adesivo sintetizado & $3,67 \mathrm{bc}^{(5,51)}$ \\
Adesivo comercial & $3,69 \mathrm{~b}^{(7,01)}$ \\
$\mathrm{HCl}-40 \mathrm{ml}$ & $4,17 \mathrm{~b}^{(7,06)}$ \\
$\mathrm{HCl}-60 \mathrm{ml}$ & $5,18 \mathrm{a}^{(7,62)}$ \\
\hline
\end{tabular}


${ }^{*}$ Médias seguidas pelas mesmas letras não diferem entre si pelo teste Tukey; $\mathrm{p}>0,05 . \mathrm{CV}=16 \%$. Parênteses $=$ desvio padrão.

Dentre os ácidos mais usuais para esse tipo de reação, segundo Kragh (2015), o ácido clorídrico é o que apresenta menor reatividade, na seguinte ordem: $\mathrm{HI}>\mathrm{HBr}>\mathrm{HCl}$, por outro lado, é o ácido menos nocivo, mais acessível em um ambiente comum de laboratório e de custo consideravelmente baixo, o que viabilizaria a sua utilização no processo de produção de adesivos.

No entanto, Santos et al. (2003) estudando adesivos produzidos a partir de creosoto desmetilado, observou que, com a desmetilação os valores de resistência das lâminas foram inferiores aquelas coladas com adesivo sintetizado em laboratório (fenol-formaldeído), o que demonstra que a desmetilação é um processo ainda passível de estudo e com grande potencial quando feito na lignina para fins de produção de adesivo, como comprovado nesse trabalho.

\section{Conclusões}

O tempo médio de gelatinização de adesivos a base de lignina desmetilada e fenolada foi $56 \%$ menor que o tempo de gelatinização do adesivo comercial. Portanto, a desmetilação é um processo com potencial de aumento da reatividade da lignina para fins de produção de adesivos. Os adesivos preparados com lignina fenolada e desmetilada com ácido clorídrico apresentam potencial de uso para colagem de lâminas de madeira. As lâminas de madeira que apresentaram maior resistência ao cisalhamento foram as coladas com adesivos produzidos com lignina desmetilada com $\mathrm{HCl}-60 \mathrm{ml}$.

\section{Referências}

ABNT - Associação Brasileira de Normas Técnicas NBR7190 - Projeto de estruturas de madeira; p. 107, 1997.

ABNT - Associação Brasileira de Normas Técnicas NBR8112, Carvão vegetal - análise imediata. 1983.
ASTM - AMERICAN SOCIETY FOR TESTING AND MATERIALS. Annual book of ASTM standards: Adhesives. Washington D. C.: 608 p., 1994.

CARROTT, P.; CARROTT, M. R. et al. Lignin-from natural adsorbent to activated carbon: A review. Bioresource Technology, v. 98, p.2301-2312, 2007.

CAVDAR, A. D.; KALAYCIOGLU, H.; HIZIROGLU, S. Some of the properties of oriented strandboard manufactured using kraft lignin phenolic resin. Journal of Materials Processing Technology, v. 202, p. 59-63, 2008.

EFFENDI, A.; GERHAUSER, H.; BRIDGWATER, A. V. Production of renewable phenolic resins by thermochemical conversion of biomass: A review, Renewable and Sustainable Energy Reviews, v. 12, p. 2092-2116, 2008.

FERHAN, M.; YAN, N.; SAIN, M. A New Method for Demethylation of Lignin from Woody Biomass using Biophysical Methods. Journal of Chemical Engineering \& Process Technology, v. 4, n. 160, 2013.

FERREIRA, D. F. Sisvar - sistema de análise de variância para dados balanceados. Lavras: UFLA, 19 p., 1998.

FERREIRA, J. C. Sínteses de adesivos de ureia-formaldeído com adição de nanocristalina, lignina Kraft e celulose. 2017. Tese de Doutorado em Ciência Florestal. Universidade Federal de Viçosa. 119 p., 2017.

GHAFFAR, S. H.; FAN, M. Lignin in straw and its applications as an adhesive. International Journal of Adhesion and Adhesives, v. 48, p. 92-101, 2014.

GOSSELINK, R. J. A.; GURAN, B.; ABACHERLI, A. Coordination network for lignin-standardisation, production and application adapted to market requirements (Eurolignin), Industrial Crops and Products, v. 20, p. 121 129, 2004.

HU, L.; PAN, H.; ZHOU, Y.; ZHANG, M. Lignin modification: mini review, BioResources, v. 6, n. 3, p. 3515 3525, 2011.

HU, L.; PAN, H.; ZHOU, Y.; HSE, C.Y.; LIU, C.; ZHANG, B.; $\mathrm{XU}, \mathrm{B}$. Chemical groups and structural characterization of lignin via thiol-mediated demethylation. Journal of Wood Chemistry and Technology, v. 34, p. 122-134, 2014.

KHAN, M.; ASHRAF, S. M.; MALHOTRA, V. P. Development and characterization of wood adhesive using bagasse lignin. International Journal of Adhesion and Adhesives, Guildford, v. 24, n. 6, p. 485 - 493, Dez, 2004. 
KRAGH, H.S. Convenções e ordem da natureza: Algumas perspectivas históricas. University of Copenhagen Amazonia, v. 11, p. 5-22, 2015.

LEE, S. H.; TERAMOTO, Y.; SHIRAISHI, N. Resol-type phenolic resin from liquefied phenolated wood and its application to phenolic foam, Journal of Applied Polymer Science, v. 84, p. $468-472,2002$.

LEI, H.; DU, G.; WU, Z.; XI, X.; DONG, Z. Cross-linked soybased wood adhesives for plywood. International Journal of Adhesion and Adhesives, v. 50, p. 199-203, 2014.

LORA, J. H.; GLASSER, W. G.; Recent application of lignin: A sustainable alternative to nonrenewable materials, Journal of Polymers and the Environment, v. 10, p. 39 - 48, 2002.

MEISTER, J.J. Polymer Modification: Principles, Techniques, and Applications: New York, p. 67-144, 2000.

PRAMANIK, S.; KARAK, N. Polymer Nanocomposites for Adhesive, Coating, and Paint Applications. In Properties and Applications of Polymer Nanocomposites, Springer Berlin Heidelberg, pp. 173-204, 2017.

THAKUR, V. K..; THAKUR, M. K..; RAGHAVAN, P.; KESSLER, M. R. Progress in green polymer composites from lignin for multifunctional applications: a review. ACS Sustainable Chemistry \& Engineering, v. 2, p. 1072-1092, 2014.

TEJADO, A.; PEÑA, C.; LABIDI, J.; ECHEVERRIA, J. M.; MONDRAGON, I. Physico-chemical characterization of lignins from different sources for use in phenolformaldehyde resin synthesis. Bioresource Technology, Espanha, v. 98, p. 1655-1663, 2007.

QIAO, W.; LI, S.; GUO, G.; HAN, S.; REN, S.; MA, Y. Synthesis and characterization of phenol-formaldehyde resin using enzymatic hydrolysis lignin. Journal of Industrial and Engineering Chemistry, v. 21, p. 1417-1422, 2015.

SANTOS, C.B.; PIMENTA, A.S.; VITAL, B.R.; BARBOSA, L.C.A. Efeito da variação do ph e da temperatura de síntese no Desempenho de adesivos à base de creosoto vegetal desmetilado-formaldeído. Revista Árvore, Viçosa, v. 27, n. 4 , p. 551-559, 2003. 